\title{
Gas and Dust in Protogalaxies
}

\author{
Francoise Combes \\ DEMIRM, Observatoire de Paris, \\ 61 Av. de l'Observatoire, F-75 014, Paris, France
}

September 1, 1999

\begin{abstract}
The study of high-redshift objects is rapidly developing, allowing to build the star formation history of the Universe. Since most of the flux from starbursts comes out in the FIR region, the submm and $\mathrm{mm}$ are privileged domains for the exploration at high z. I review the recent work on galaxies at high redshift in this wavelength region, for the continuum as well as for the line detection (dust and molecular gas). Perspectives are discussed to detect early objects (maybe protogalaxies) with the future large millimeter instruments.
\end{abstract}

Keywords: molecules, dust, galaxies, millimeter

\section{Introduction}

Considerable progress has been made in our knowledge of galaxy evolution from the faint galaxy studies made possible by HST deep imaging, ground-based spectroscopy, and wide faint galaxy surveys (e.g. Ellis, 1998; Steidel et al. 1996; Shade et al. 1996). This progress has been led by the optical domain, but crucial information came also from the far-infrared and millimeter domains: the cosmic IR and submm background radiation discovered by COBE (e.g. Puget et al. 1996; Hauser et al. 1998) yields an insight on the global past star-formation of the Universe, and the sources discovered at high redshift in the millimeter continuum and lines yield information on the structure of the past starbursts (Smail et al. 1997; Guilloteau et al. 1999). From all these data, a global view of star formation as a function of lookback time has been derived (e.g. Madau et al. 1996; Glazebrook et al. 1999), which still is submitted to big uncertainties, especially at high redshift. In particular, it is possible that the optically derived star formation rate is under-estimated, due to dust obscuration, and that only infrared/submm surveys could give the correct information (Guiderdoni et al. 1997).

This review focus on the dust and molecular content of galaxies, as a way to trace the evolution of star formation, and to detect the location of starbursts as a function of redshift. First the present state of knowledge is detailed, concerning $\mathrm{CO}$ emission lines as well as dust continuum, and their interpretation is discussed (respective role of starburst and AGN for instance). Then perspectives are drawn concerning

(c) 2018 Kluwer Academic Publishers. Printed in the Netherlands. 
the future surveys that will be conducted with the next generation of millimeter instruments.

\section{Detection of Molecular Gas at High Redshift}

\subsection{EMISSION LINES}

The detection of high-redhifted $(z>2)$ millimeter $\mathrm{CO}$ lines in the hyperluminous object IRAS 10214+4724 $(z=2.28$, Brown \& Vanden

Bout 1992, Solomon et al. 1992), has opened a new way of research to tackle the star formation history of the Universe. Although the object turned out to be highly gravitationally amplified, it revealed however that galaxies at this epoch could have large amounts of molecular gas, excited by an important starburst, and sufficiently metal-enriched to emit detectable CO emission lines. The latter bring fundamental information about the cold gas component in high-z objects and therefore about the physical conditions of the formation of galaxies and the first generations of stars. At high enough redshifts, most of the galaxy mass could be molecular. The main problem to detect this molecular component could be its low metallicity, but theoretical calculations have shown that in a violent starburst, the metallicity could reach solar values very quickly (Elbaz et al. 1992).

After the first discovery, many searches for other candidates took place, but they were harder than expected, and only a few, often gravitationally amplified, objects have been detected: the lensed Cloverleaf quasar $\mathrm{H} 1413+117$ at $z=2.558$ (Barvainis et al. 1994), the lensed radiogalaxy MG0414+0534 at $z=2.639$ (Barvainis et al. 1998), the possibly magnified object BR1202-0725 at $z=4.69$ (Ohta et al. 1996, Omont et al. 1996a), the amplified submillimeter-selected hyperluminous galaxies SMM02399-0136 at $z=2.808$ (Frayer et al. 1998), and SMM $14011+0252$ at 2.565 (Frayer et al. 1999), and the magnified BAL quasar APM08279+5255, at $z=3.911$, where the gas temperature derived from the CO lines is $\sim 200 \mathrm{~K}$, maybe excited by the quasar (Downes et al. 1999a). Recently Scoville et al. (1997b) reported the detection of the first non-lensed object at $z=2.394$, the weak radio galaxy 53W002, and Guilloteau et al. (1997) the radio-quiet quasar BRI 1335-0417, at $z=4.407$, which has no direct indication of lensing. If the non-amplification is confirmed, these objects would contain the largest molecular contents known $\left(8-10 \cdot 10^{10} \mathrm{M}_{\odot}\right.$ with a standard $\mathrm{CO} / \mathrm{H}_{2}$ conversion ratio, and even more if the metallicity is low). The derived molecular masses are so high that $\mathrm{H}_{2}$ would constitute between 30 to $80 \%$ of the total dynamical mass (according to the unknown 


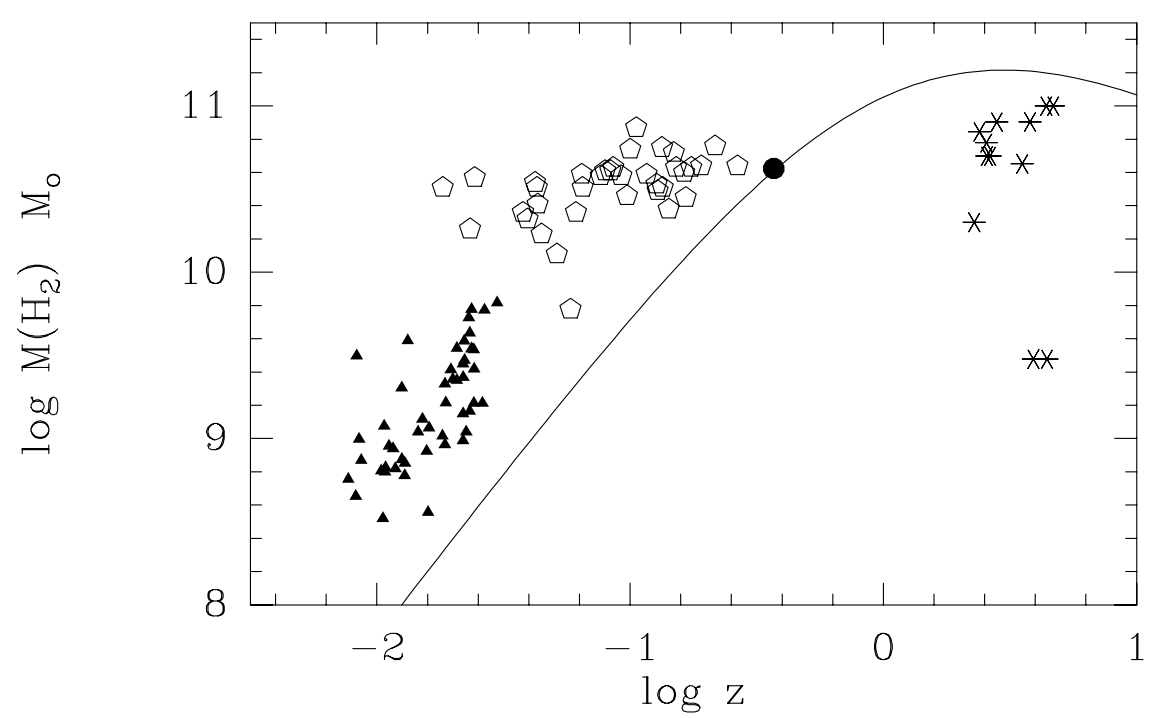

Figure 1. $\mathrm{H}_{2}$ masses for the CO-detected objects at high redshift (stars), compared to the ultra-luminous-IR sample of Solomon et al. (1997, open pentagons), to the Coma supercluster sample from Casoli et al (1996, filled triangles), and to the quasar 3c48, marked as a filled dot (Scoville et al. 1993, Wink et al. 1997). The curve indicates the $3 \sigma$ detection limit of $\mathrm{I}(\mathrm{CO})=1 \mathrm{~K} \mathrm{~km} / \mathrm{s}$ at the IRAM-30m telescope (equivalent to an rms of $1 \mathrm{mK}$, with an assumed $\Delta V=300 \mathrm{~km} / \mathrm{s}$ ). Note the absence of detected objects between 0.36 and 2.2 in redshift, where the sensitivity is insufficient, and the gravitational lenses maybe not yet frequent enough to compensate. The points at high $z$ can be detected well below the sensitivity limit, since they are gravitationally amplified.

inclination), if the standard $\mathrm{CO} / \mathrm{H}_{2}$ conversion ratio was adopted. The application of this conversion ratio is however doubtful, and it is possible that the involved $\mathrm{H}_{2}$ masses are 3-4 times lower (Solomon et al. 1997).

The CO line detections at high $z$ up to now are summarized in Table I, and the molecular masses as a function of redshift are displayed in Fig. 1. It is clear from this figure that our present sensitivity prevents detection of $\mathrm{CO}$ lines above a redshift of 0.4, unless the objects are lensed; but this will rapidly change with the new millimeter instruments planned over the world (the Green-Bank-100m of NRAO, the LMT-50m of UMass-INAOE, the ALMA (Europe/USA) and the LMSA (Japan) interferometers). It is therefore interesting to predict with simple models the detection capabilities, as a function of redshift, metallicity or physical conditions in the high-z objects. In particular, it would be highly interesting to detect not only the few exceptional 
amplified monsters in the sky, but also the widely spread normal galaxy population of the young universe. A previous study of galaxies at very high redshift (up to $z=30$ ) by Silk \& Spaans (1997) concluded that $\mathrm{CO}$ lines could be even more easy to detect than the continuum; The models presented in section 4 do not agree with this conclusion.

Today galaxies are detected in the optical up to $z=6$, when the age of the universe is about $5 \%$ of its age, or $10^{10} \mathrm{yr}$ in a standard flat universe model. For larger redshifts, it is likely that the total amount of cumulated star formation is not a significant fraction of the total (e.g. Madau et al. 1996). However, it is of overwhelming interest to trace the first star-forming structures, as early as possible to constrain theories of galaxy formation.

Table I. CO data for high redshift objects

\begin{tabular}{lcccccc}
\hline Source & $z$ & $\mathrm{CO}$ & $\mathrm{S}$ & $\Delta \mathrm{V}$ & $\begin{array}{c}\mathrm{MH}_{2} \\
\mathrm{mJy}\end{array}$ & $\mathrm{Ref}$ \\
& & $\mathrm{km} / \mathrm{s}$ & $10^{10} \mathrm{M}_{\odot}$ & \\
\hline F10214+4724 & 2.285 & $3-2$ & 18 & 230 & $2^{*}$ & 1 \\
53W002 & 2.394 & $3-2$ & 3 & 540 & 7 & 2 \\
H 1413+117 & 2.558 & $3-2$ & 23 & 330 & $2-6^{*}$ & 3 \\
SMM 14011+0252 & 2.565 & $3-2$ & 13 & 200 & $5^{*}$ & 4 \\
MG 0414+0534 & 2.639 & $3-2$ & 4 & 580 & $5^{*}$ & 5 \\
SMM 02399-0136 & 2.808 & $3-2$ & 4 & 710 & $8^{*}$ & 6 \\
6C1909+722 & 3.532 & $4-3$ & 2 & 530 & 4.5 & 7 \\
4C60.07 & 3.791 & $4-3$ & 1.7 & 1000 & 8 & 7 \\
APM 08279+5255 & 3.911 & $4-3$ & 6 & 400 & $0.3^{*}$ & 8 \\
BR 1335-0414 & 4.407 & $5-4$ & 7 & 420 & 10 & 9 \\
BR 0952-0115 & 4.434 & $5-4$ & 4 & 230 & $0.3^{*}$ & 10 \\
BR 1202-0725 & 4.690 & $5-4$ & 8 & 320 & 10 & 11 \\
\hline
\end{tabular}

* corrected for magnification, when estimated Masses have been rescaled to $H_{0}=75 \mathrm{~km} / \mathrm{s} / \mathrm{Mpc}$. When multiple images are resolved, the flux corresponds to their sum

(1) Solomon et al. (1992), Downes et al. (1995); (2) Scoville et al. (1997b); (3) Barvainis et al. (1994, 1997); (4) Frayer et al. (1999); (5) Barvainis et al. (1998); (6) Frayer et al. (1998); (7) Papadopoulos et al. (1999); (8) Downes et al. (1999a); (9) Guilloteau et al. (1997); (10) Guilloteau et al. (1999); (11) Omont et al. (1996a)

\subsection{Absorption Lines}

Molecular absorption lines are also a powerful tool to study the interstellar medium of galaxies at high redshift (e.g. Combes \& Wiklind 1996). A sample of the molecular lines detected is shown in Fig. 2. 

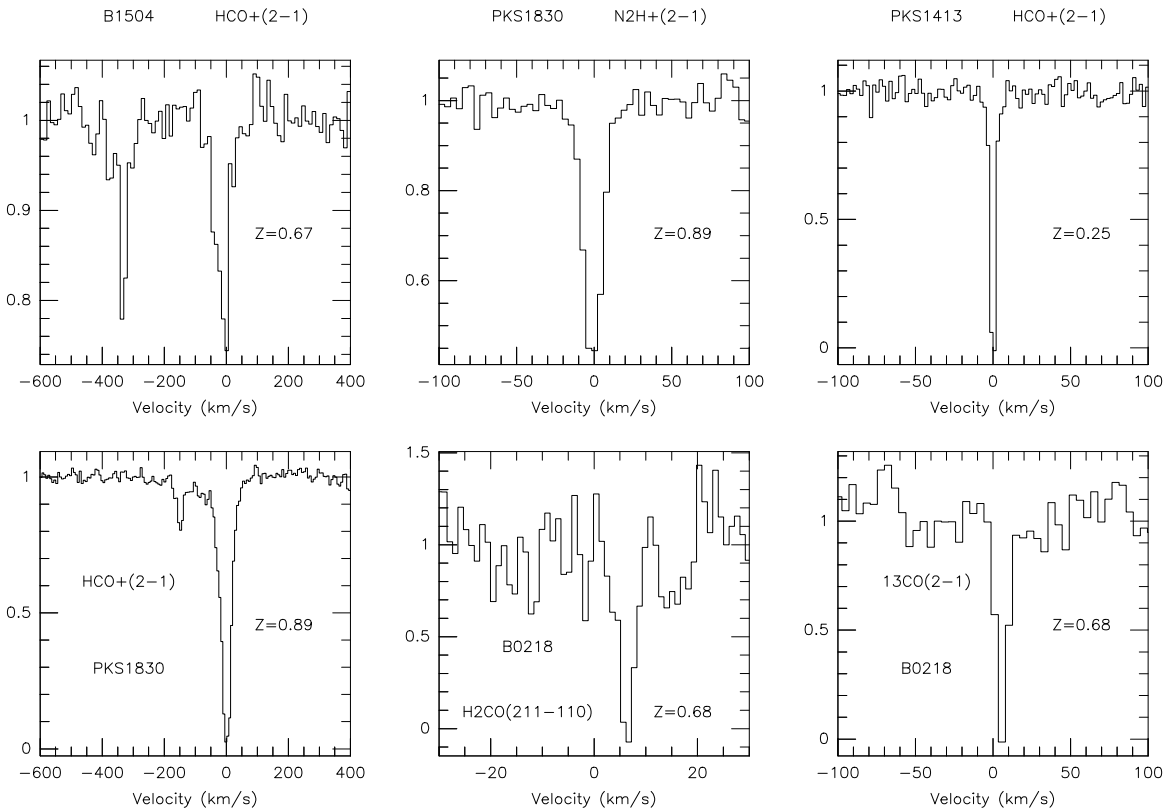

Figure 2. Examples of molecular absorption lines detected in the millimeter range. Lines can be extremely narrow (below $1 \mathrm{~km} / \mathrm{s}$ ) up to quite broad $(100 \mathrm{~km} / \mathrm{s})$. More than 20 different molecules or transitions have been detected in one single absorption system. Here the continuum sources are B3 1504+377 (Wiklind \& Combes 1996b), PKS 1830-211 (Wiklind \& Combes 1996a), PKS 1413+357 (Wiklind \& Combes 1997) and B0218+357 (Wiklind \& Combes 1995). The signal has been normalised to the continuum level detected.

These molecular absorption objects are the continuation at high column densities $\left(10^{21}-10^{24} \mathrm{~cm}^{-2}\right)$ of the whole spectrum of absorption systems, from the Ly $\alpha$ forest $\left(10^{12}-10^{19} \mathrm{~cm}^{-2}\right)$ to the damped Ly $\alpha$ and HI $21 \mathrm{~cm}$ absorptions $\left(10^{19}-10^{21} \mathrm{~cm}^{-2}\right)$. It is currently thought that the $\operatorname{Ly} \alpha$ forest originates from gaseous filaments in the extra-galactic medium, that the damped and HI absorptions involve mainly the outer parts of spiral galaxies. The molecular absorptions concern the central parts of galaxies. The properties of molecular absorptions detected in the millimeter domain so far, are summarised in Table II.

The utility of molecular absorption lines comes from the high sensitivity. Due to the small extent of the background continuum source, the signal is not diluted, there is no distance dependence. Molecular absorption lines are as easy to detect at $\mathrm{z} \approx 1$ as at $\mathrm{z} \approx 5$, provided the continuum sources exist. Then, direct opacity are measured, and it is almost as easy to detect many high dipole molecules, $\left(\mathrm{HCO}^{+}\right.$or $\left.\mathrm{HCN}\right)$ as CO. 
Table II. Properties of molecular absorption line systems in the $\mathrm{mm}$

\begin{tabular}{lccccccc} 
Source & $\mathrm{z}_{\mathrm{a}}^{a}$ & $\mathrm{z}_{\mathrm{e}}^{b}$ & $\begin{array}{c}N_{\mathrm{CO}} \\
\mathrm{cm}^{-2}\end{array}$ & $\begin{array}{c}N_{\mathrm{H}_{2}} \\
\mathrm{~cm}^{-2}\end{array}$ & $\begin{array}{c}N_{\mathrm{HI}}^{e} \\
\mathrm{~cm}^{-2}\end{array}$ & $\begin{array}{c}\mathrm{A}_{V}^{\prime c} \\
N_{\mathrm{HI}} / N_{H_{2}}\end{array}$ \\
\hline Cen-A & 0.00184 & 0.0018 & $1.0 \cdot 10^{16}$ & $2.0 \cdot 10^{20}$ & $1.0 \cdot 10^{20}$ & 50 & 0.5 \\
3C 293 & 0.0446 & 0.0446 & $\geq 3.0 \cdot 10^{16}$ & $\geq 6.0 \cdot 10^{20}$ & $1.2 \cdot 10^{21}$ & - & $\geq 0.5$ \\
PKS1413+357 & 0.24671 & 0.247 & $2.3 \cdot 10^{16}$ & $4.6 \cdot 10^{20}$ & $1.3 \cdot 10^{21}$ & 2.0 & 2.8 \\
B3 1504+377A & 0.67335 & 0.673 & $6.0 \cdot 10^{16}$ & $1.2 \cdot 10^{21}$ & $2.4 \cdot 10^{21}$ & 5.0 & 2.0 \\
B3 1504+377B & 0.67150 & 0.673 & $2.6 \cdot 10^{16}$ & $5.2 \cdot 10^{20}$ & $<7 \cdot 10^{20}$ & $<2$ & $<1.4$ \\
B 0218+357 & 0.68466 & 0.94 & $2.0 \cdot 10^{19}$ & $4.0 \cdot 10^{23}$ & $4.0 \cdot 10^{20}$ & 850 & $1 \cdot 10^{-3}$ \\
PKS1830-211A & 0.88582 & 2.51 & $2.0 \cdot 10^{18}$ & $4.0 \cdot 10^{22}$ & $5.0 \cdot 10^{20}$ & 100 & $1 \cdot 10^{-2}$ \\
PKS1830-211B & 0.88489 & 2.51 & $1.0 \cdot 10^{16 d}$ & $2.0 \cdot 10^{20}$ & $1.0 \cdot 10^{21}$ & 1.8 & 5.0 \\
PKS1830-211C & 0.19267 & 2.51 & $<6 \cdot 10^{15}$ & $<1 \cdot 10^{20}$ & $2.5 \cdot 10^{20}$ & $<0.2$ & $>2.5$ \\
\hline
\end{tabular}

${ }^{a}$ Redshift of absorption line ${ }^{b}$ Redshift of background source ${ }^{c}$ Extinction corrected for redshift using a Galactic extinction law ${ }^{d}$ Estimated from the $\mathrm{HCO}^{+}$column density of $1.3 \cdot 10^{13} \mathrm{~cm}^{-2}$ e $21 \mathrm{~cm}$ HI data taken from Haschick \& Baan (1985, 3C293) and Carilli et al. 1992, 1993, 1998

About 15 different molecules have been detected in absorption at high redshifts, in a total of 30 different transitions. This allows a detailed chemical study and comparison with local clouds. Within the large dispersion in column densities, and in molecular cloud properties, the high redshift systems do not appear to be different from local ones, suggesting that the conditions for star formation are the same up to $\mathrm{z} \sim 1$ as at the present.

A systematic survey for absorption lines has also been undertaken in front of about a hundred continuum sources candidates, selected from flat-spectrum continuum sources. The continuum needs to be at least $0.2 \mathrm{Jy}$ to allow detection of intervening molecular gas. The redshift of the absorbing candidate is known, either from previously detected $\mathrm{HI}$ absorption, or from optical lines emission. When the continuum source is strong enough, at least $1 \mathrm{Jy}$, and no redshift is known, it is possible to search for absorption lines by scanning in frequency (cf. Wiklind \& Combes 1996a). This last method is the most promising with the new generation millimeter instruments, that will gain an order of magnitude in sensitivity. Indeed, the best candidates are the most obscured ones, where no redshift is available.

A lot more absorption systems could be found with the future instruments, at faint continuum flux, since the number counts of quasars are a non-linear function of flux and the local luminosity function is steep 
(see Peacock 1985). However, the flat-spectrum radio-loud quasars distribution is decreasing sharply at $z>3$ (Shaver et al. 1996), and this is not likely due to obscuration, since they are radio-selected quasars. If quasars are associated to galaxy formation and interactions, this tends to show that the decrease of star-formation rate beyond $z=3$ is real and not an extinction effect. The relative absence of strong continuum radio sources at high redshift will not help the tracing of protogalaxies through absorption techniques.

\section{Detection of Dust at High Redshift}

\subsection{Submm CONTINUUm SURVEYS}

The spectral energy distribution (SED) of galaxies over the radio, $\mathrm{mm}$ and FIR domains has a characteristic maximum around 60-100 $\mu \mathrm{m}$ due to dust heated by newly born stars, and the interstellar radiation field (see Fig. 3). This maximum depends on the dust temperature, and the curve is that of a grey-body, where the optical thickness can be modelled by a power-law in frequency, $\tau \propto \nu^{\beta}$, where $\beta \sim 1.5-2$, according to the nature of dust. In the Rayleigh-Jeans domain (frequencies lower than the maximum), the flux increases almost as $\nu^{4}$, and this creates what is called a negative K-correction, i.e. it begins to be more easy to detect objects at high redshift than low redshift, at a given frequency, and sky surveys could be dominated by remote objects (see e.g. Blain \& Longair 1993, 1996). The source counts could be inverted in favor of high-redshift objects, if they exist in equal numbers (not depopulated by strong evolution effects). The millimeter domain becomes then a privileged tool to tackle galaxy formation.

In Fig. 3, we see that at low frequency (lower than $100 \mathrm{GHz}$, or $3 \mathrm{~mm}$ in wavelength), the radio spectrum is due to synchrotron processes. There is a marked flux minimum that can be used as a redshift indicator (e.g. Carilli \& Yun 1999; Blain 1999). Indeed, there is a well-known tight correlation between the synchrotron radio power and the far-infrared emission in star-forming galaxies (Condon 1992). This correlation is thought to arise because both FIR and non-thermal radio emission are both directly proportionnal to recent star-formation (the FIR being a

good measure of massive stars luminosity, and the radio of the rate of supernovae). However, the z-indicator is somewhat ambiguous, since there is a degeneracy between increasing the redshift or decreasing the dust temperature.

The bulk of the high redshift galaxies presently known have been discovered in optical. But these surveys could have missed dust-enshrouded 


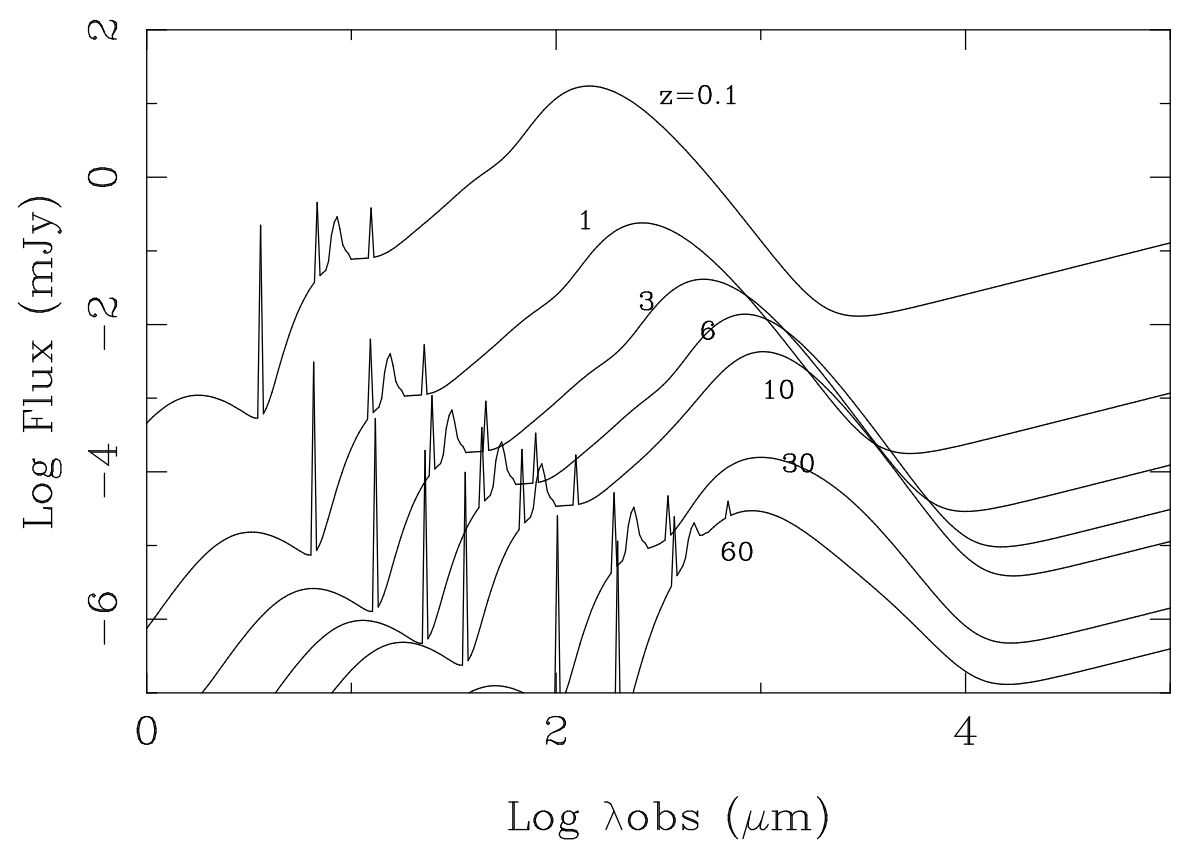

Figure 3. Spectral flux distribution for a typical ULIG starburst source in the radio and far-infrared, for various redshifts $z=0.1,1,3,6,10,30,60\left(H_{0}=75 \mathrm{~km} / \mathrm{s} / \mathrm{Mpc}\right.$, $\left.q_{0}=0.5\right)$. At right is a synchrotron spectrum, in a power-law of slope -0.7 , and left the emission from dust, modelled by PAHs, very small grains and big grains, as in Désert et al. (1990) to fit the Milky Way data. It has been assumed here that the dust properties are the same as in our Galaxy, and that the power of the starburst is the same at any redshift, i.e. $T_{\mathrm{dust}}^{6}-T_{\mathrm{bg}}^{6}$ is conserved.

starbursts, since we now know that dust and high metallicity occur very early in the universe (cf. the previous sections). Submm and FIR deep surveys are then the best strategy to detect starbursting proto-galaxies, and many such works have been undertaken, either with sensitive array-bolometers on single dishes (IRAM-30m, SCUBA on JCMT, ...) mm-interferometers, or with ISOPHOT and ISOCAM on board of ISO satellite.

The first deep search was made with the SCUBA bolometer (Holland et al. 1999) towards a cluster of galaxies, thought to serve as a gravitational lens for high- $z$ galaxies behind (Smail et al. 1997). The amplification is in average a factor 2 . This has the combined advantage to increase the sensitivity, and to reduce the source confusion, since there should be little contamination from cluster galaxies (Blain 1997). A large number of sources were found, all at large redshifts $(z>1)$, extrapolated to 2000 sources per square degree (above $4 \mathrm{mJy}$ ), revealing a large positive evolution with redshift, i.e. an increase of starbursting 
galaxies. Searches toward the Hubble Deep Field-North (Hughes et al. 1998), and towards the Lockman hole and SSA13 (Barger et al. 1998), have also found a few sources, allowing to derive a similar density of sources: 800 per square degree, above $3 \mathrm{mJy}$ at $850 \mu \mathrm{m}$. This already can account for $50 \%$ of the cosmic infra-red background (CIRB), that has been estimated by Puget et al. (1996) and Hauser et al. (1998) from COBE data. The photometric redshifts of these sources range between 1 and 3. Their identification with optical objects might be uncertain (Richards 1999). However, Hughes et al. (1998) claim that the star formation rate derived from the far-infrared might be in some cases 10 times higher than derived from the optical, due to the high extinction.

Eales et al. (1999) surveyed some of the CFRS fields at $850 \mu \mathrm{m}$ with SCUBA and found also that the sources can account for a significant fraction of the CIRB background $(\sim 30 \%)$. Their interpretation in terms of the star formation history is however slightly different, in that they do not exclude that the submm luminosity density could evolve in the same way as the UV one. Deep galaxy surveys at 7 and $15 \mu \mathrm{m}$ with ISOCAM also see an evolution with redshift of star-forming galaxies: heavily extincted starbursts represent less than $1 \%$ of all galaxies, but $18 \%$ of the star formation rate out to $z=1$ (Flores et al. 1999).

Now that a few dozens of submm sources have been catalogued (Barger et al. 1999a, Smail et al. 1999), the count rates are confirmed, i.e. $\sim 1000$ source per square degree, above $3 \mathrm{mJy}$, at $850 \mu \mathrm{m}$, and even 8000 above $1 \mathrm{mJy}$, from the gravitationally amplified cluster fields (Blain et al. 1999a). The cumulative count rate can be fitted by a powerlaw, above $2 \mathrm{mJy}$, with a slope of -2.2 . The main difficulty appears to be the identification of the submm sources with optical or radio counterparts: the spatial resolution of the submm surveys are several arcsecs, with sometimes systematic uncertainties, and some of the previous claimed identifications have been reconsidered (e.g. Barger et al. 1999b, Downes et al. 1999b). Follow-up in the radio (CO lines) or nearinfrared, or optical to find redshifts, are much slower than the surveys themselves. At least $20 \%$ of the sources reveal an AGN activity, and the bulk of the sources are at relatively low redshift $1<z<3$ (Barger et al. 1999a).

\subsection{EXTREMELy RED OBJECTS, EROs}

To search for primeval galaxies, already Elston et al. (1988) had identified extremely red objects that are conspicuous only in the nearinfrared, and have R - K colors larger than 5. The ERO class could include several categories of objects: essentially old stellar populations at high redshift, or high- $z$ dust-reddened starbursts (Hu \& Ridgway 
1994; Cowie et al. 1996). Maybe $10 \%$ of the submm sources could be EROs (Smail et al. 1999). A proto-typical ERO at $z=1.44$ (Dey et al. 1999) has been detected in submm continuum (Cimatti et al. 1998), and has been found to be an ultraluminous starburst shrouded by dust, with a star formation rate of $200-500 \mathrm{M}_{\odot}$ per yr. The surface density of such EROs at $\mathrm{K}<20$ and color $\mathrm{R}-\mathrm{K}>6$ is about 500 per square degree, and for $\mathrm{R}-\mathrm{K}>7$, about 50 per square degree (which is comparable to the number of QSO with $\mathrm{B}<21.5$, Andreani et al. 1999). At least for the few objects known, the AGN cannot be the source of the huge luminosity, given the FIR/radio ratio.

\subsection{AGN OR StarbURSTS}

For a long time, the highest redshift objects known were quasars, although now, with higher sensitivities, the situation is reversed (e.g. $\mathrm{Hu}$ et al. 1998). Surveys have therefore been done in the millimeter continuum and lines for high-z quasars (McMahon et al. 1994; Isaak et al. 1994; Omont et al. 1996a, 1996b; Guilloteau et al. 1999). Results have shown that the far-infrared luminosities of many of them are due to dust heated by a starburst, although the AGN activity is simultaneously present. But since the manifestations of the two nuclear activities (starburst or AGN) are in many cases similar, and are most of the time associated, it has become a controversial question to disentangle the two interpretations. Ultra-luminous starbursts take place over the few central 100pc (Solomon et al. 1997), and even pure AGN activities can be mimicked by radio supernovae (Boyle \& Terlevich 1998). Genzel et al. 1998 from ISO mid-infrared diagrams concluded that the ultra-luminous galaxies are powered at 30\% from AGN and $70 \%$ from star formation; a similar conclusion is suggested by Cooray \& Haiman (1999) for submm catalogued sources. McMahon et al. (1999) however suggest that a much more significant fraction of submm sources, between 15 to $100 \%$, could be AGN-powered. Note that distinction criteria are hard to find (cf. Stein 1995), and even symbiotic starburstblack hole models are likely (Williams et al. 1999). Discriminating between the two possibilities can have important consequences on the star formation history of the Universe.

\subsection{Star Formation Rate}

One of the breakthrough due to recent progress on faint galaxies has been the inventory of the amount of star formation at every epoch (e.g. Madau et al. 1996). The comoving star formation rate is increasing like $(1+z)^{4}$ from $z=0$ to $z=1$, and then decreases again to the same present value down to $z=5$. But this relies on the optical 
studies, i.e. on the UV-determined star forming rates in the rest-frame. If early starbursts are dusty, this decrease could be changed into a plateau (Guiderdoni et al. 1997, 1998; Blain et al. 1999b). Since the AGN-starbursts nature of the submm sources is still an open question, Trentham et al. (1999) consider two extreme possibilities: either the submm faint galaxies are all dusty starbursts, but even then, they do not dominate the star formation rate in the Madau plot at any redshift; or they are dusty AGN, and again they cannot represent more than a few percents of the present density of dark objects, as inferred by Magorrian et al. (1998).

\section{Perspectives with Future mm Instruments}

If progenitors of quasars or protogalaxies form at high redshifts (larger than 10), then the millimeter domain is the best place to detect them (Loeb 1993; Braine 1995). Both continuum and line emission could be detected, provided enough sensitivity, with about 10 times more collecting surface as present ones (either with a single dish, as the GBT$100 \mathrm{~m}$ of NRAO, or with the ALMA interferometer). As was clear in the previous sections, the detection of the submm continuum emission from actively star-forming objects at high redshifts is much easier than the $\mathrm{CO}$ line detection. The line emission does not have such a negative Kcorrection, since in the low frequency domain, the flux of the successive lines increases roughly as $\nu^{2}$ (optically thick domain), instead of $\nu^{4}$ for the continuum. Nevertheless the line emission is essential to study the nature of the object (the AGN-starburst connection for instance), and deduce more physics (kinematics, abundances, excitation, etc..). Given the gas and dust temperatures, the maximum flux is always reached at much lower frequencies than in the continuum, since the lines always reflect the energy difference between two levels; this is an advantage, given the largest atmospheric opacity at high frequencies.

\subsection{Predicted Line And Continuum Fluxes}

The best tracer of molecular gas at large-scale is the CO molecule, the most abundant after $\mathrm{H}_{2}$. All other molecules will give weaker signals. The fine-structure CII line at $158 \mu \mathrm{m}$, formed in PDR at the border of molecular clouds, is also thought to be a useful tool for proto-galaxies or proto-quasars (Loeb 1993), but it has revealed disapointing in starbursts, or compact objects, due to optical thickness or inefficiency of gas heating (e.g. Malhotra et al. 1997): the $\mathrm{L}_{\mathrm{CII}} / \mathrm{L}_{\mathrm{FIR}}$ ratio decreases as $\mathrm{L}_{\mathrm{FIR}} / \mathrm{L}_{\mathrm{B}}$ increases. 
To model high-redshift starburst objects, let us extrapolate the properties of more local ones: the active region is generally confined to a compact nuclear disk, sub-kpc in size (Scoville et al. 1997a, Solomon et al. 1990, 1997). The gas is much denser here than in average over a normal galaxy, of the order of $10^{4} \mathrm{~cm}^{-3}$, with clumps at least of $10^{6} \mathrm{~cm}^{-3}$ to explain the data on high density tracers (HCN, CS..); large gas masses can pile up in the center, due to torques exerted in galaxy interactions and mergers (e.g. Barnes \& Hernquist 1992, 1996). To schematize, the ISM maybe modelled by two density and temperature components, at 30 and $90 \mathrm{~K}$ (cf. Combes et al. 1999). The total molecular mass considered will be $6 \cdot 10^{10} \mathrm{M}_{\odot}$ and the average column density $\mathrm{N}\left(\mathrm{H}_{2}\right)$ of $10^{24} \mathrm{~cm}^{-2}$, typical of the Orion cloud center.

Going towards high redshift $(z>9)$, the temperature of the cosmic background $\mathrm{T}_{\mathrm{bg}}$ becomes of the same order as the interstellar dust temperature, and the excitation of the gas by the background radiation competes with that of gas collisions. It might then appear easier to detect the lines (Silk \& Spaans 1997), but this is not the case when every effect is taken into account. To have an idea of the increase of the dust temperature with $z$, the simplest assumption is to consider the same heating power due to the starburst. At a stationary state, the dust must then radiate the same energy in the far-infrared that it receives from the stars, and this is proportional to the quantity $T_{\text {dust }}^{6}-T_{\mathrm{bg}}^{6}$, if the dust is optically thin, and its opacity varies in $\nu^{\beta}$, with $\beta=2$. Keeping this quantity constant means that the energy re-radiated by the dust, proportional to $T_{\text {dust }}^{6}$, is always equal to the energy it received from the cosmic background, proportional to $T_{\mathrm{bg}}^{6}$, plus the constant energy flux coming from the stars. Since $\beta$ can also be equal to 1 or 1.5 , or the dust be optically thick, we have also considered the possibility of keeping $T_{\text {dust }}^{4}-T_{\text {bg }}^{4}$ constant; this does not change fundamentally the results.

Computing the populations of the CO rotational levels with an LVG code, and in the case of the two component models described earlier, the predictions of the line and continuum intensities as a function of redshift and frequencies are plotted in Fig. 4.

When comparing these predictions with the present instrumental sensitivities, it appears that the continuum is detectable at any redshift already, for an ultra-luminous source, while the line emission has to await the order of magnitude increase that will be provided by the next generation in the $\mathrm{mm}$ and sub-mm domains. The recent reported detections (cf. Table I) have been possible because of gravitational lens magnifications (or maybe for 1-2 cases, an exceptional object). 

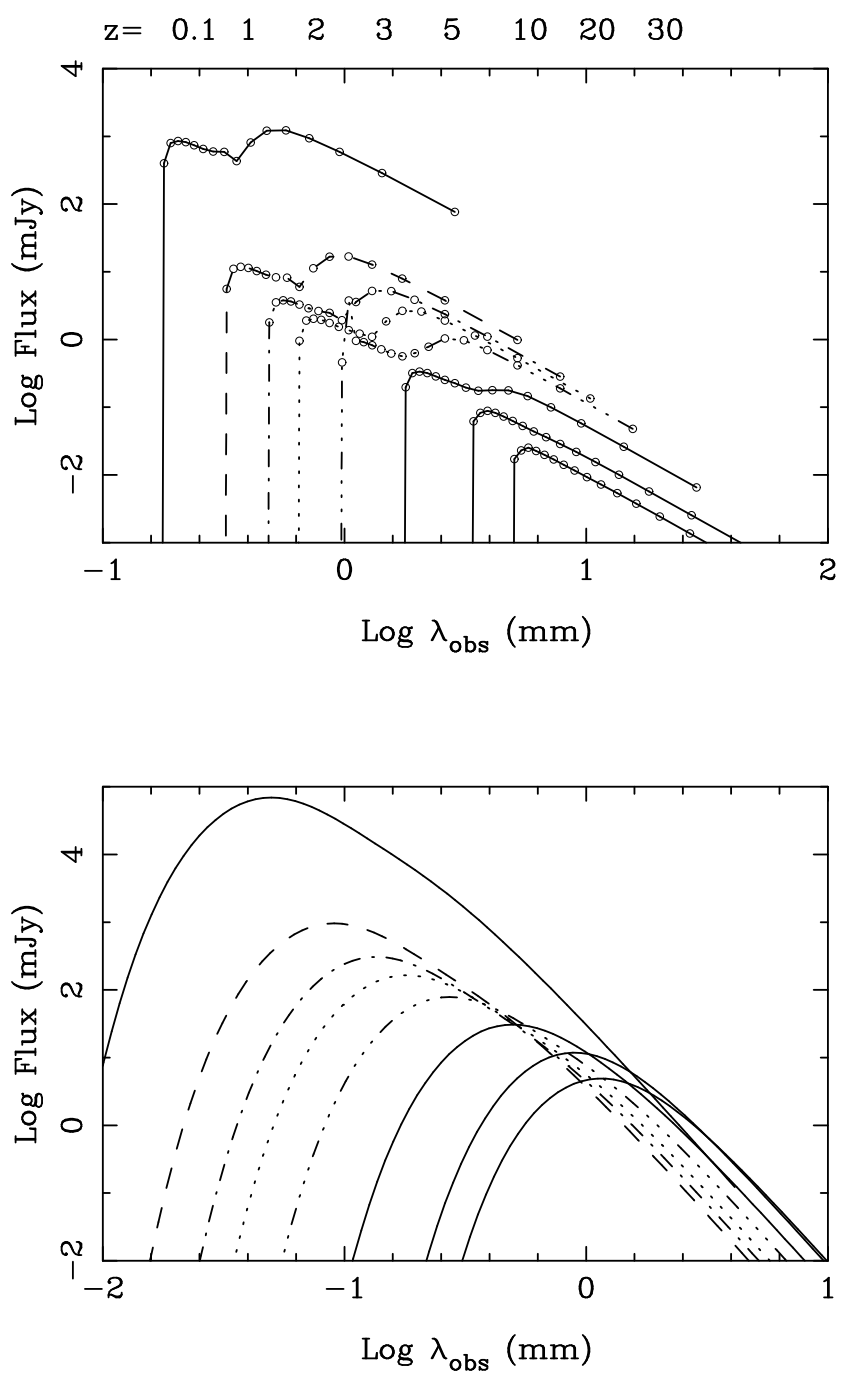

Figure 4. Expected flux for the two-component cloud model, for various redshifts $z$ $=0.1,1,2,3,5,10,20,30$, and $q_{0}=0.5$. Top are the CO lines, materialised each by a circle (they are joined by a line only to guide the eye). Bottom is the continuum emission from dust. It has been assumed here that $T_{\text {dust }}^{6}-T_{\mathrm{bg}}^{6}$ is conserved (from Combes et al. 1999).

\subsection{Source Counts}

To predict the number of sources that will become available with the future sensitivity, let us adopt a simple model of starburst formation, in the frame of the hierarchical theory of galaxy formation. The cosmology adopted here is an Einstein-de Sitter model, $\Omega=1$, with no 
cosmological constant, and $H_{0}=75 \mathrm{~km} / \mathrm{s} / \mathrm{Mpc}, q_{0}=0.5$. The number of mergers as a function of redshifts can be easily computed through the Press-Schechter formalism (Press \& Schechter 1974), assuming selfsimilarity for the probability of dark halos merging (i.e. independent of mass, Blain \& Longair 1993): the mass spectrum of bound objects is, at any redshift $z$ :

$$
\frac{d N}{d M_{P S}} \propto M^{-2}\left(\frac{M}{M^{*}}\right)^{\gamma / 2} \exp \left[-\left(\frac{M}{M^{*}}\right)^{\gamma}\right]
$$

where $\gamma=(3+n) / 3$, and $n$ the power-law slope of the primordial fluctuations $(n=-1$ here, so that $\gamma=2 / 3)$. The turn-off mass $M^{*}$ is increasing with redshift as:

$$
M^{*} \propto(1+z)^{-2 / \gamma}
$$

Following Blain et al. (1999c), we also write the merger rate as a function of $M$ and $z$ under the form:

$$
\frac{d}{d t} \frac{d N}{d M_{P S}}+\phi \frac{d N}{d M_{P S}}\left(\frac{-2}{\gamma} \frac{d z}{d t(1+z)}\right) \exp \left[(1-\alpha)\left(\frac{M}{M^{*}}\right)^{\gamma}\right]
$$

This shape (with $\phi=1.7$ and $\alpha=1.4$ ) has been chosen so that the merger rate is the sum of the net evolution of the Press-Schechter distribution at any mass, plus a specific term, indicating that the merger probability is maximum at equal masses, and is exponentially vanishing at high mass ( cf. Blain \& Longair 1993).

This gives the number of mergers at each epoch, but the efficiency of mergers in terms of star-formation must also vary considerably with redshift, with a peak at $z \sim 2$, to agree with the observations (such as in Fig. 5). Also, the integration over all redshifts of the flux of all sources should agree with the cosmic infrared background detected by COBE (Fig. 5). These contribute to reduce the number of free parameters of the modelling. To fit the source counts, however, another parameter must be introduced which measures the rate of energy released in a merger (or the life-time of the event): this rate must increase strongly with redshift (cf. Blain et al. 1999c). Once the counts are made compatible with the submm observations, the model indicates what must be the contributions of the various redshift classes to the present counts (cf. Fig. 5). It is interesting to note that the intermediate redshifts dominate the counts $(2<z<5)$, if we allow the star formation to begin before $z=6$. At higher dust temperature, the counts are dominated by the highest redshifts $(z>5)$. But these contributions depend strongly on the adopted shape of the star-forming efficiency versus $z$. Observations of these counts and their redshift distribution will therefore bring a lot of insight in the physiscs of early protogalaxies. 

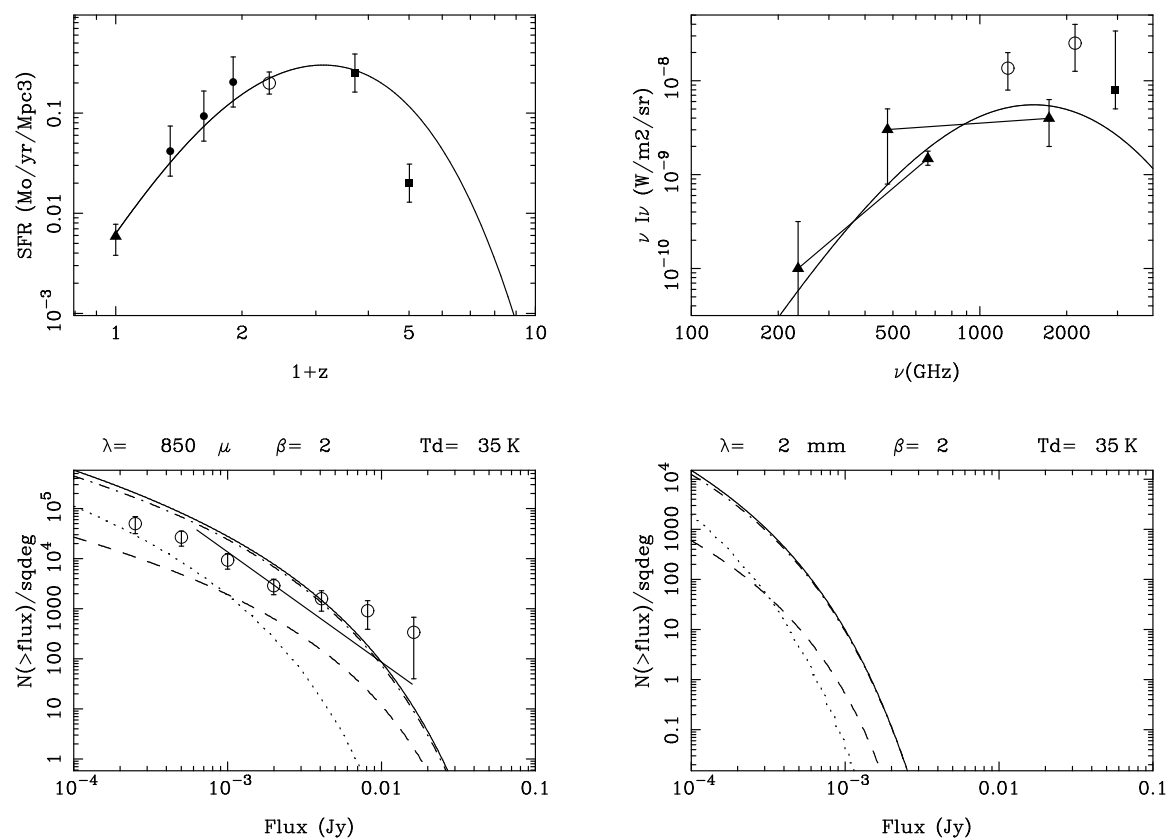

Figure 5. Top left: History of the star formation rate, adopted by the hierarchical model used here, compared with observations. The point at $z=0$ is from Gallego et al. (1995), the 3 following ones (full circles) from Flores et al. (1999), the empty circle from Yan et al. (1999), and the two high redshift points (filled squares), from Pettini et al. (1998). Top right: Predictions of the model for the far-infrared cosmic background: the observations have been used to constrain the parameters of the model. The filled triangles with a line joining them symbolise the spectrum derived by Puget et al. (1996) from COBE, the filled circles are from Hauser et al. (1998), and the filled square from Dwek et al. (1998). Bottom left and right: Source counts predicted at $850 \mu \mathrm{m}$ and $2 \mathrm{~mm}$ respectively: the solid line is the total. Dash is the lowest redshifts $(z<2)$; Dot-dash, intermediate $(2<z<5)$; Dots are the highest redshifts $(z>5)$. The emission from the dusty starbursts have been assumed a grey-body at a temperature of $35 \mathrm{~K}$, and an opacity varying as $\nu^{2}$. The empty circles are data from Blain et al. (2000), and the straight line is a fit to the data derived by Barger et al. (1999a) at $850 \mu \mathrm{m}$.

It is possible to derive also the source counts for the CO line emission. The spectral energy distribution is now obtained with a comb-like function, representing the rotational ladder, convolved with a Planck distribution of temperature equal to the dust temperature, assuming the lines optically thick. The frequency filling factor is then proportional to the rotational number, and therefore to the redshift, for a given observed frequency. The width of the lines have been assumed to be $300 \mathrm{~km} / \mathrm{s}$. The derived source numbers are shown in Fig. 6 . Note how they are also dominated by the high redshift sources. It is however not 


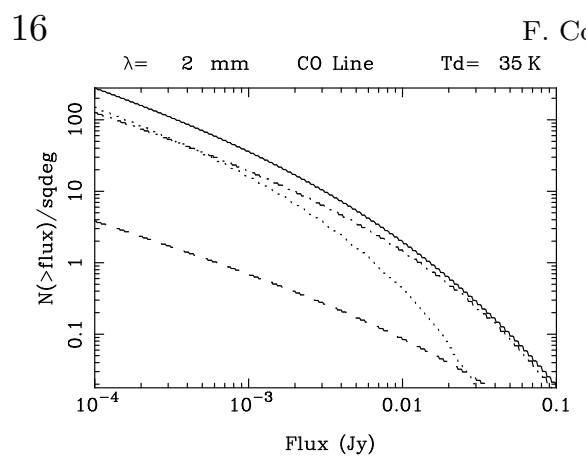

F. Combes

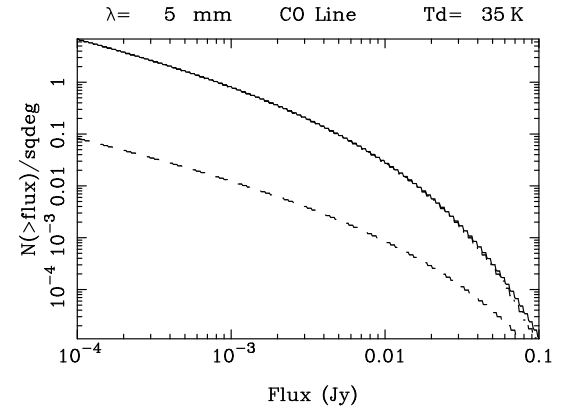

Figure 6. Left Source counts for the CO lines at an observed frequency of $2 \mathrm{~mm}$, assuming optically thick gas at $\mathrm{T}_{e x}=35 \mathrm{~K}$. Right Same for $\lambda=5 \mathrm{~mm}$. The meanings of the line styles are as in fig 5

useful to observe at $\lambda$ below $1 \mathrm{~mm}$ for high- $z$ protogalaxies, but instead to shift towards $\lambda=1 \mathrm{~cm}$.

\section{Conclusion}

If there were as many ultra-luminous starbursts at $z=1$ than at $z=5$, the present sub-mm continuum surveys should have detected them (there might still be identification problems, however). A good way to identify them is to search for molecular lines, but the present instruments are not yet sensitive enough, when they are not helped by gravitational lenses. With more than an order of magnitude sensitivity that will be available in the next decade, it will become possible to detect normal star-forming galaxies at high redshift, and even protogalaxies at $z>5$.

\section{References}

Andreani P., Cimatti A., Röttgering H., Tilanus R.: in Proceedings of the Workshop 'Ultraluminous Infrared Galaxies: Monsters or Babies', Ringberg Castle, Germany, Sep. 1998 (astro-ph/9903121)

Barger, A.J., Cowie, L.L., Sanders, et al.: 1998, Nature, 394, 248

Barger, A.J., Cowie, L.L., Sanders, D.B.: 1999a, ApJ 518, L5

Barger, A.J., Cowie, L.L., Smail I. et al. : 1999b, AJ 117, 2656

Barnes J.E., Hernquist L.: 1992, ARA\&A 30, 705

Barnes, J.E., \& Hernquist, L., 1996, ApJ, 471, 115

Barvainis R., Alloin D., Guilloteau S., Antonucci R. 1998, ApJ 492, L13

Barvainis R., Tacconi L., Antonucci R., Coleman P.: 1994, Nature 371, 586

Blain A.W.: 1997, MNRAS 290, 553

Blain A.W.: 1999, MNRAS in press (astro-ph/9906438)

Blain A.W., Ivison R.J., Kneib J-P., Smail I.: 2000, in Proceedings of "The Hyredshift Universe", eds. A.J. Bunker, W.J.M. van Breugel, (astro-ph/9908024) 
Blain A.W., Jameson A., Smail I. et al. : 1999c, MNRAS in press (astro-ph/9906311)

Blain A.W., Kneib J-P., Ivison R.J., Smail I.: 1999a, ApJ 512, L87

Blain A.W., Longair M.S.: 1993, MNRAS 264, 509

Blain A.W., Longair M.S.: 1996, MNRAS 279, 847

Blain A.W., Smail I., Ivison R.J., Kneib J-P.: 1999b, MNRAS 302, 632

Boyle, B. J., Terlevich, R. J.: 1998, MNRAS 293, L49

Braine J.: 1995, A\&A 300, 20

Brown R., Vanden Bout P.: 1992, ApJ 397, L19

Carilli, C.L., Menten K.M., Reid M.J., Rupen, M.P., Yun M.S.: 1998, ApJ 494, 175

Carilli C.L., Perlman E.S., Stocke J.T.: 1992, ApJ 400, L13

Carilli, C.L., Rupen, M.P., Yanny, B. 1993, ApJ 412, L59

Carilli, C.L., Yun M.S.: 1999, ApJ 513, L13

Casoli F., Dickey J., Kazes I. et al.: 1996, A\&AS 116, 193

Cimatti A., Andreani P., Röttgering H., Tilanus R.: 1998, Nature 392, 895

Combes F., Maoli R., Omont A.: 1999, A\&A 345, 369

Combes F., Wiklind T.: 1996, in "Cold gas at high redshift", ed. Breme r M., Rottgering H., van der Werf P., Carilli C.L. (Dordrecht:Kluwer), p. 215

Condon, J.J.: 1992, ARAA 30, 575

Cooray A.R., Haiman Z.: 1999, BAAS, 194.2007

Cowie, L. L., Songaila, A., Hu, E. M., Cohen, J.G.: 1996, AJ 112, 839

Désert F-X., Boulanger F., Puget J-L.: 1990, A\&A 237, 215

Dey, A., Graham, J. R., Ivison, R. J. et al.: 1999, ApJ 519, 610

Downes D., Neri R., Greve A., et al.: 1999b, A\&A 347, 809

Downes D., Neri R., Wiklind T., Wilner D.J., Shaver P.: 1999a, ApJ 513, L1

Downes D., Solomon P.M., Radford S.J.E. 1995, ApJ 453, L65

Dwek E. et al. : 1998, ApJ 508, 106

Eales, S., Lilly, S., Gear, et al.: 1999, ApJ 515, 518

Elbaz D., Arnaud M., Casse M., et al.: 1992, A\&A 265, L29

Ellis R., 1998, Nature, 395, A3

Elston R., Rieke G.H., Rieke M.J.: 1988, ApJ 331, L77

Evans, A. S., Sanders, D. B., Surace, J. A., Mazzarella, J. M.: 1999, ApJ 511, 730

Flores H. et al. : 1999, ApJ 517, 148

Frayer D.T., Ivison R.J., Scoville N.Z., et al., 1998, ApJ 506, L7

Frayer D.T., Ivison R.J., Scoville N.Z., et al., 1999, ApJ 514, L13

Gallego J., Zamorano J., Aragón-Salamanca A., Rego M.: 1995, ApJ 455, L1 (erratum ApJ 459, L43)

Genzel, R., Lutz, D., Sturm, E. et al., 1998, ApJ, 498, 579

Glazebrook K., Blake C., Economou F., Lilly S., Colless M.: 1999, MNRAS 306, 843

Guiderdoni B., et al.: 1997, Nature 390, 257 1997, A\&A, 328, L1

Guiderdoni B., Hivon E., Bouchet F.R., Maffei B.: 1998 MNRAS 295, 877

Guilloteau S., Omont A., Cox P., McMahon R.G., PetitJean P.: 1999, A\&A preprint

Guilloteau S., Omont A., McMahon R.G., Cox P., PetitJean P.: 1997, A\&A 328, L1

Haschick, A. D., Baan, W. A.: 1985, ApJ 289, 574

Hauser, M. et al., 1998, ApJ 508, 25

Holland W.S., Robson E.I., Gear W.K, et al.: 1999, MNRAS 303, 659

Hu, E.M., Cowie L.L., McMahon, R.G.: 1998, ApJ, 502 L99

Hu, E. M., Ridgway, S. E.: 1994, AJ 107, 1303

Hughes D.H., Serjeant S., Dunlop J. et al.: 1998, Nature 394, 241

Isaak, K.G., McMahon, R.G., Hills, R.E., Withington, S., 1994, MNRAS, 269, L28

Loeb A.: 1993, ApJ 404, L37

Madau, P., Ferguson, H. C., Dickinson, M. E., et al. : 1996, MNRAS 283, 1388 
Magorrian, J., et al., 1998, AJ, 115, 2285

Malhotra, S., Helou, G., Stacey, G., et al.:1997, ApJ 491, L27

McMahon R.G., Omont A., Bergeron J., Kreysa E., Haslam C.G.T., 1994, MNRAS, 267, L9

McMahon R.G., Priddey R.S., Omont A., Snellen I., Withington S., 1999, MNRAS, in press (astro-ph/9907239)

Ohta K., Yamada T., Nakanishi K., et al.: 1996, Nature 382, 426

Omont A., McMahon R.G., Cox P., et al.: 1996b, A\&A 315, 1

Omont A., Petitjean P., Guilloteau S., McMahon R.G., Solomon P.M.: 1996a, Nature 382,428

Papadopoulos P.P., Rottgering H.J.A., van der Werf P.P., et al. 1999, ApJ, preprint (astro-ph/9908286)

Peacock J.A.: 1985, MNRAS 217, 601

Pettini M., Kellog M., Steidel C.C. et al.: 1998, ApJ 508, 539

Press W.H., Schechter P.: 1974, ApJ 187, 425

Puget, J.-L., Abergel, A., Bernard, J.-P., et al. : 1996, A\&A 308, L5

Richards E.R.: 1999, ApJ 513, L9

Shaver P.A., Wall J.V., Kellermann K.I., Jackson C.A., Hawkins M.R.S.: 1996, Nature 384, 439

Scoville N.Z., Padin S., Sanders D.B. et al. : 1993, ApJ 415, L75

Scoville N.Z., Yun M.S., Bryant P.M.: 1997a, ApJ 484, 702

Scoville N.Z., Yun M.S., Windhorst R.A., Keel W.C., Armus L.: 1997b, ApJ 485, L21

Schade, D., Lilly, S. J., Le Fevre, O., Hammer, F., Crampton, D.: 1996, ApJ 464, 79

Silk J., Spaans M.: 1997, ApJ 488, L79

Solomon P.M., Downes D., Radford S.J.E.: 1992, Nature 356, 318

Solomon P.M., Downes D., Radford S.J.E., Barrett J.W.: 1997, ApJ 478, 144

Solomon P.M., Radford S.J.E., Downes D.: 1990, ApJ 348, L53

Smail, I., Ivison, R.J., Blain, A.W., 1997, ApJ, 490, L5

Smail, I., Ivison, R.J., Kneib, J-P., et al.: 1999, MNRAS in press (astro-ph/9905246)

Steidel, C. C., Giavalisco, M., Pettini, M., Dickinson, M., Adelberger, K. L.: 1996, ApJ 462, L17

Stein W.A.: 1995, AJ 110, 1019

Trentham N., Blain A.W., Goldader G.: 1999, MNRAS 305, 61

Williams R.J.R., Baker A.C., Perry J.J.: 1999, MNRAS in press (astro-ph/9907198)

Wiklind T., Combes F.: 1995, A\&A 299, 382

Wiklind T., Combes F.: 1996a, Nature 379, 139

Wiklind T., Combes F.: 1996b, A\&A 315, 86

Wiklind T., Combes F.: 1997, A\&A 328, 48

Wink J.E., Guilloteau S., Wilson T.L., 1997, A\&A 322, 427

Yan L., McCarthy P.J., Freudling W. et al.:1999, ApJ 519, L47 